\title{
Editorial NPL 1/2019
}

\author{
Jens Ivo Engels • Volker Köhler
}

Online publiziert: 6. Februar 2019

(C) Gesellschaft zur wissenschaftlichen Förderung politischer Literatur e.V. and the Author(s) 2019

\section{Liebe Leserinnen und Leser,}

Sie haben es gemerkt: ,Ihre“ „Neue Politische Literatur“ sieht jetzt anders aus. Ab diesem 64. Jahrgang erscheinen die „Berichte aus Geschichts- und Politikwissenschaft" in neuem Gewand und bei einem neuen Verlag, nämlich Springer VS.

Der Wechsel ist ein tiefer Einschnitt, nachdem wir knapp drei Jahrzehnte im Hause Peter Lang verlegt wurden. In dieser langen Zeit hat sich die Verlagslandschaft stark gewandelt. Wir sind überzeugt, dass der Wechsel für uns alle - für Leser- und Autorenschaft, aber auch Redaktion - Vorteile mit sich bringt. Allerdings geschieht dies durchaus im Zeichen der Kontinuität: Nach wie vor werden wir dreimal jährlich unser bewährtes gedrucktes Heft mit Sammel- und Einzelrezensionen herausbringen. Gleichzeitig wird unsere Online-Präsenz gestärkt. Dank des neuen publish online first-Verfahren werden unsere Beiträge nach Erteilung der Druckfreigabe online gestellt - und zwar jeweils einzeln, sobald sie fertig gestellt sind. Durch die DOI werden die Beiträge sofort eindeutig identifizier- und zitierbar. Die „Neue Politische Literatur" kann somit schneller und flexibler an wissenschaftlichen Debatten teilnehmen. Im Printformat werden die Beiträge nach wie vor zusammengeführt.

Auf diese Weise wollen wir unsere alten Qualitäten behalten und durch neue (digitale) Stärken ergänzen. Unser Anspruch bleibt bestehen, Ihnen eine forschungszentrierte und kritische Literaturschau in der Neuesten Geschichte, Ideengeschichte und Politischen Theorie anzubieten.

\footnotetext{
J. I. Engels $(\bowtie)$

Institut für Geschichte, Technische Universität Darmstadt, Dolivostraße 15, 64293 Darmstadt, Deutschland

E-Mail: engels@pg.tu-darmstadt.de
} 
Im vorliegenden Heft finden Sie eine Sammelrezension zur Bedeutung der Nationalismusforschung für die Politikwissenschaft (Julia Schulze Wessel), eine Sammelbesprechung zum Konzept des Rechtspluralismus (Eva Weiler/Andreas Niederberger), einen Rezensionsaufsatz zur Wissenschaftsgeschichte (Fabian Link) und einen Essay zu Neuerscheinungen im 50. Jubiläumsjahr der Revolte von 1968 (Werner Bührer).

Wir würden uns freuen, wenn wir Sie mit diesem Heft und auch in Zukunft digital wie analog durch die geschichts- und politikwissenschaftliche Forschung begleiten dürfen. Bevor Sie nun in unserem Heft zu stöbern beginnen, sei uns eine letzte Anmerkung gestattet: Wir möchten auch auf diesem Weg unserem Herausgeber Jürgen Osterhammel gratulieren. Er hat 2018 den Balzan-Preis für seine herausragenden Arbeiten auf dem Gebiet der Globalgeschichte erhalten. Wir freuen uns mit ihm.

Mit herzlichen Grüßen

Jens Ivo Engels

Volker Köhler 